Published in final edited form as:

Mult Scler. 2015 June ; 21(7): 866-874. doi:10.1177/1352458514555785.

\title{
Antibodies to MOG and AQP4 in adults with neuromyelitis optica and suspected limited forms of the disease
}

\author{
Romana Höftberger\# \\ Center of Neuroimmunology, Service of Neurology, Hospital Clínic and Institut d'Investigació \\ Biomèdica August Pi i Sunyer (IDIBAPS), Universitat de Barcelona, Barcelona, Spain/Institute of \\ Neurology, Medical University of Vienna, Austria

\begin{abstract}
María Sepulveda\#
Center of Neuroimmunology, Service of Neurology, Hospital Clínic and Institut d'Investigació Biomèdica August Pi i Sunyer (IDIBAPS), Universitat de Barcelona, Barcelona, Spain
\end{abstract}

\section{Thaís Armangue and Yolanda Blanco} \\ Center of Neuroimmunology, Service of Neurology, Hospital Clínic and Institut d'Investigació \\ Biomèdica August Pi i Sunyer (IDIBAPS), Universitat de Barcelona, Barcelona, Spain

\section{Kevin Rostásy} \\ Center of Neuroimmunology, Service of Neurology, Hospital Clínic and Institut d'Investigació \\ Biomèdica August Pi i Sunyer (IDIBAPS), Universitat de Barcelona, Barcelona, Spain/Department \\ of Pediatrics I, Division of Pediatric Neurology, Innsbruck Medical University, Innsbruck, Austria/ \\ Pediatric Neurology, Witten/Herdecke University, Children's Hospital Datteln, Germany
}

\author{
Alvaro Cobo Calvo \\ Servicio de Neurología, Hospital Universitari de Bellvitge, IDIBELL, L'Hospitalet de Llobregat, \\ Barcelona, Spain
}

Javier Olascoaga

Hospital Universitario Donostia, San Sebastián, Spain

\section{Lluís Ramió-Torrentà}

Hospital Universitari Dr. Josep Trueta, IDIBGI, Girona, Spain

\author{
Markus Reindl \\ Clinical Department of Neurology, Innsbruck Medical University, Innsbruck, Austria \\ Julián Benito-León
}

\footnotetext{
Reprints and permissions: http://www.sagepub.co.uk/journalsPermissions.nav

Correspondence to: Albert Saiz, Service of Neurology, Hospital Clínic. Villarroel 170, 08036 Barcelona, Spain. asaiz@ clinic.ub.es. Conflict of interest Höftberger, Sepúlveda, Armangue, Blanco, Cobo Calvo, Reindl, Benito-León, Casanova, Sabater, and Graus report no disclosures. Rostásy has received compensation for consulting services and speaking Merck-Serono, Biogen-Idec; Olascoaga has received compensation for consulting services and speaking from Bayer-Schering, Biogen - Idec, Merck-Serono, Novartis, SanofiGenzyme and Teva Pharmaceutical Industries Ltd; Ramió-Torrentà has received compensation for speaking from Bayer-Healthcare, Biogen-Idec, Merck-Serono, Novartis, Teva Pharmaceutical Industries Ltd; received research support from Bayer-Healthcare, BiogenIdec, Merck-Serono, and Teva Pharmaceutical Industries Ltd; serves on scientific advisory board for Biogen-Idec; Arrambide has received reimbursements of travel expenses for scientific meetings from Merck-Serono; Dalmau has a research grant from Euroimmun, and receives royalties from patents for the use of Ma2 and NMDAR as autoantibody tests; Saiz has received compensation for consulting services and speaking from Bayer-Schering, Merck-Serono, Biogen-Idec, Sanofi-Aventis, Teva Pharmaceutical Industries Ltd and Novartis
} 
Departament of Neurology, University Hospital "12 de Octubre", Centro de Investigación Biomédica en red sobre Enfermedades Neurodegenerativas (CIBERNED), Departamento de Medicina, Complutense University, Madrid, Spain

\section{Bonaventura Casanova}

Hospital Universitari La Fe de Valencia, Valencia, Spain

\section{Georgina Arrambide}

Servei de Neurologia-Neuroimunologia, Centre d'Esclerosi Múltiple de Catalunya (Cemcat), Vall d'Hebron Institut de Recerca, Hospital Universitari Vall d'Hebron, Universitat Autònoma de Barcelona, Barcelona, Spain

\section{Lidia Sabater and Francesc Graus}

Center of Neuroimmunology, Service of Neurology, Hospital Clínic and Institut d'Investigació Biomèdica August Pi i Sunyer (IDIBAPS), Universitat de Barcelona, Barcelona, Spain

\section{Josep Dalmau}

Center of Neuroimmunology, Service of Neurology, Hospital Clínic and Institut d'Investigació Biomèdica August Pi i Sunyer (IDIBAPS), Universitat de Barcelona, Barcelona, Spain/Institució Catalana de Recerca i Estudis Avançats (ICREA), Barcelona, Spain

\section{Albert Saiz}

Center of Neuroimmunology, Service of Neurology, Hospital Clínic and Institut d'Investigació Biomèdica August Pi i Sunyer (IDIBAPS), Universitat de Barcelona, Barcelona, Spain

\# These authors contributed equally to this work.

\section{Abstract}

Objective-We aimed to report the frequency and implications of antibodies to myelin oligodendrocyte glycoprotein (MOG-ab) in adults with demyelinating syndromes suspicious for neuromyelitis optica (NMO).

Methods-Samples from 174 patients (48 NMO, 84 longitudinally extensive myelitis (LETM), 39 optic neuritis (ON), and three acute disseminated encephalomyelitis (ADEM) who presented initially with isolated LETM) were retrospectively examined for AQP4-ab and MOG-ab using cell-based assays.

Results-MOG-ab were found in 17 (9.8\%) patients, AQP4-ab in 59 (34\%), and both antibodies in two (1.1\%). Among the 17 patients with MOG-ab alone, seven (41\%) had ON, five (29\%) LETM, four (24\%) NMO, and one (6\%) ADEM. Compared with patients with AQP4-ab, those with MOG-ab were significantly younger (median: 27 vs. 40.5 years), without female predominance (53\% vs. $90 \%$ ), and the clinical course was more frequently monophasic (41\% vs. 7\%) with a benign outcome (median Expanded Disability Status Scale: 1.5 vs. 4.0). In eight patients with paired serum-cerebrospinal fluid (CSF) samples, five had MOG-ab in both samples and three only in serum. Antibody titres did not differ among clinical phenotypes or disease course. MOG-ab remained detectable in 12/14 patients (median follow-up: 23 months) without correlation between titres' evolution and outcome. 
Conclusion-MOG-ab identify a subgroup of adult patients with NMO, LETM and ON that have better outcome than those associated with AQP4-ab. MOG-ab are more frequently detected in serum than CSF and the follow-up of titres does not correlate with outcome.

\section{Keywords}

Neuromyelitis optica; longitudinally extensive myelitis; optic neuritis; aquaporin-4 antibody; antibodies to myelin oligodendrocyte glycoprotein

\section{Introduction}

Aquaporin 4 antibodies (AQP4-ab) are useful biomarkers for neuromyelitis optica (NMO) and limited forms of the disease, including longitudinally extensive transverse myelitis (LETM) and recurrent optic neuritis (ON). ${ }^{1}$ Up to $30 \%$ of patients with NMO are AQP4-ab negative, ${ }^{2,3}$ it being unclear whether this group represents a different clinical entity. ${ }^{4}$ Recently, antibodies to myelin oligodendrocyte glycoprotein (MOG-ab) were described in patients, usually children, with demyelinating syndromes.5 In children, MOG-ab are present in monophasic demyelinating diseases, mainly acute disseminated encephalomyelitis (ADEM),${ }^{6-8}$ and less frequently in NMO and recurrent ON. ${ }^{9,10}$ Children with NMO and MOG-ab seem to have a more favourable outcome than those with AQP4-ab. ${ }^{8,9}$ Whether similar prognostic implications apply to adults with MOG-ab is currently unclear because the number of studies is limited and all of them included a mixed population of children and adults. ${ }^{11-13}$ The aim of the present study was to determine the frequency and implications of MOG-ab in adults with NMO and demyelinating syndromes suspicious for NMO.

\section{Methods}

\section{Patients}

Between November 2005 and November 2013 we examined 2348 serum samples of patients with demyelinating disorders sent to our laboratory to test AQP4-ab. We selected those that fulfilled these inclusion criteria: (1) age of the patient $\geq 18$ years; (2) a definitive diagnosis of NMO according to Wingerchuk diagnostic criteria of 1999 or $2006,{ }^{14,15}$ LETM, ${ }^{16}$ severe (visual acuity $<20 / 200$ ) or bilateral or recurrent $\mathrm{ON}^{1}{ }^{17,18}$ and (3) available stored samples. Most of the samples, along with a standardized questionnaire with the clinical data, were referred by neurologists of the Spanish Group of Neuromyelitis optica ${ }^{18}$ and the Spanish Network of Multiple Sclerosis (Red Española de Esclerosis Múltiple, REEM). ${ }^{16}$ Positivity for MOG-ab was retrospectively determined. When outcome data were requested, the corresponding neurologists provided the information blinded to MOG-ab testing result. Relapses were defined as a new neurological symptoms lasting at least $24 \mathrm{~h}$ and accompanied by new neurological examination findings. Brain and spinal cord magnetic resonance imaging (MRI) features were not centrally reviewed. Overall, 174 adult patients were identified, including 48 with NMO, 84 LETM, 39 ON, and three patients who presented with isolated clinical findings of transverse myelitis and presence of LETM in the spinal cord MRI, but whose subsequent clinical evolution led to a final diagnosis of ADEM. ${ }^{19}$ All but seven patients were Caucasian. Thirty paediatric patients consecutively studied during the same period (four NMO, seven LETM, eight ON, 10 ADEM, and one 
patient who developed a relapse of LETM 2 years after being diagnosed with multiple sclerosis (MS)) were used to compare the frequency of antibodies in both age groups. To evaluate the specificity of MOG-ab, sera of 55 adults with MS according to the McDonald criteria ${ }^{20}$ (20 with clinically isolated syndrome, 15 with relapsing-remitting MS, 10 with secondary progressive MS, and 10 with primary progressive MS) were included in the study. Serum and cerebrospinal fluid (CSF) samples used in the study are deposited in the collection of biological samples named "Neuroinmunología" registered in the Biobank of Institut d' Investigació Biomèdica August Pi i Sunyer (IDIBAPS), Barcelona, Spain. Informed consent for storage and use of these samples for research purposes was obtained from all patients. The study was approved by the Ethics Committee of the Hospital Clínic of Barcelona, Spain.

\section{Cell-based assays}

All samples were examined for AQP4-ab using an in-house cell-based assay (CBA) with live HEK293 cells transfected with the aquaporin-4-M23 isoform as previously reported ${ }^{21}$ (for more details see the Supplementary Material). MOG-ab were examined using an in-house CBA with HEK293 cells transfected with the full-length MOG C-terminally fused to EGFP as described previously ${ }^{22}$ (Supplementary Material and Supplementary Figure S-1). The titre of MOG-ab was obtained by serial dilutions of samples, starting at 1:160 for serum ${ }^{5}$ and 1:2 for CSF until the reactivity was no longer visible. Intrathecal synthesis of MOG-ab and AQP4-ab was evaluated as described previously. ${ }^{23}$ To confirm the simultaneous presence of MOG- and AQP4-ab in two patients, serum samples were immunoabsorbed with pellets of HEK293 cells transfected with AQP4 or with MOG. Briefly, three P60 plates of confluent HEK293 cells transfected with the appropriate plasmid were scraped and spun down at 500 $g$ for $5 \mathrm{~min}$. The pellets were resuspended and gently homogenized in DMEM (Invitrogen, Carlsbard, CA, USA) plus 10\% FCS. Sera were diluted and incubated for $2 \mathrm{~h}$ at room temperature with the previous mix. A cleared supernatant was obtained by centrifugation at $10,000 \mathrm{~g}$ for $10 \mathrm{~min}$ before the immunofluorescence assay.

\section{Statistical analysis}

Clinical data between groups were compared using nonparametric tests (Mann-Whitney $U$ test) and the categorical data were analysed with Fisher's exact test and Chi-square test when appropriate. In patients with follow-up samples we analysed the association of titre change (a decrease of at least two serial dilutions or seronegative conversion between the first and the last sample) with monophasic course or outcome (Expanded Disability Status Scale score, EDSS, $\leq 2.0$ ) with Fisher's exact test. Statistical significance was defined as two-sided $p$-value less than 0.05. The software used was IBM SPSS Statistics v19.

\section{Results}

MOG-ab were detected in 17/174 (9.8\%) adult patients, AQP4-ab in 59 (34\%) and both antibodies in two (1.1\%). Seven of the 17 (41\%) patients with isolated MOG-ab had ON, five (29\%) LETM, four (24\%) NMO, and one (6\%) ADEM (Table 1). The frequency of MOG-ab and AQP4-ab varied according to the clinical phenotype: MOG-ab occurred less frequently than AQP4-ab in NMO (8.7\% vs. $80 \%, p<0.001)$ and LETM (6\% vs. $19 \%, p=$ 
$0.017)$, and were similarly frequent in $\mathrm{ON}(18 \%$ vs. $15 \%, p=1.0)$. In comparison, paediatric patients had a similar frequency of antibodies but with a predominance of MOG-ab found in 12 patients (one also with AQP4-ab) whereas only one patient had AQP4-ab. The most common clinical phenotype was ADEM, diagnosed in $36 \%$ of paediatric patients. Control adult MS patients were MOG-ab negative.

The demographic and clinical features of MOG-ab or AQP4-ab seropositive and seronegative patients are shown in Table 1. The two patients with both antibodies presented with a classic NMO clinical picture of simultaneous bilateral ON and LETM and were excluded from analysis. Patients with isolated MOG-ab were different from those with AQP4-ab with respect to predominance of women (53\% vs. $90 \%$ female, $p=0.002)$, age at onset (median 27 vs. $40.5 \mathrm{y}, p=0.017)$, monophasic course ( $41 \%$ vs. $7 \%, p=0.002)$, use of chronic therapy (35\% vs. $91 \%, p<0.001)$ and disability at the last follow-up (median EDSS, 1.5 vs. $4.0, p<0.001$ ). Patients with MOG-ab were also different from seronegative patients with respect to age at onset (median 27 vs. $37.5 \mathrm{y}, p=0.021$ ) and disability at last visit (median EDSS 1.5 vs. 3.0, $p<0.001$ ). The clinical features of each patient with MOG-ab are shown in Table 2.

Patients received similar acute treatments regardless of antibody status. All but one patient (94\%) with isolated MOG-ab were treated with IV methylprednisolone (IVMP) (1 g/d for 35 days) and four of them (24\%) additionally underwent plasma exchange (PLEX). Similarly, $50 / 59(84.7 \%)$ of the AQP4-ab positive and 70/86 (82\%) of the seronegative patients received IVMP as acute first-line treatment ( $p=0.44$ and $p=0.29$, respectively). The percentage of patients that additionally underwent PLEX was also similar: 11/59 (19\%) of the AQP4-ab positive $(p=0.73)$ and $13 / 86(15 \%)$ of the seronegative patients $(p=0.47)$ (Table 1).

Representative cases including the two NMO patients harbouring both antibodies are described in the supplemental material: eAppendix.

\section{Comparison of MOG-ab positive patients according to clinical phenotypes}

MOG-ab were detected in 4/9 (44\%) of NMO and 5/84 (6\%) of LETM patients without AQP4-ab (Table S-1 and S-2 in supplemental material). NMO patients with MOG-ab compared with those with AQP4-ab had a better outcome with a significant lower EDSS at the last visit (median EDSS 1.8 vs. 4.0, $p=0.023$ ), despite both groups having a similar relapsing course (Table S-1 in supplemental material). LETM patients with MOG-ab compared with those with AQP4-ab were younger (median 36 vs. $46 \mathrm{y}, p=0.05$ ), and they had a more frequent monophasic course $(80 \%$ vs. $14 \%, p=0.017)$. Compared with seronegative patients, those with LETM and positive MOG-ab had a higher frequency of involvement of all spinal cord regions ( $40 \%$ vs. $3 \%, p=0.029)$ and a lower disability (median EDSS 2.0 vs. 4.0, $p=0.043$ ). Although the severity of the initial episode of LETM was similar in patients with MOG-ab, AQP4-ab and seronegative cases, the outcome of patients with MOG-ab was better (Table S-2 in supplemental material). In fact, on follow-up imaging, in 7/8 (87\%) MOG-ab patients with NMO and LETM, the spinal cord MRI showed a complete resolution (in 5) or reduction of the lesion to $<2$ vertebral segments (in 2), a 
feature only observed in 4/20 (20\%) of AQP4-ab positive patients $(p=0.002)$ and $8 / 23$ $(35 \%)$ of seronegative patients $(p=0.016)$.

MOG-ab were found in 7/39 (18\%) of ON patients, accounting for $21 \%$ of the seronegative AQP4-ab patients (Table S-3 in supplemental material). MOG-ab patients compared with those with AQP4-ab had a higher frequency of relapses and lower disability, although because of the small numbers the differences were not significant $(p=0.058$ and $p=0.052$, respectively), and compared with seronegative patients had a significant lower disability (median EDSS 1.0 vs. 3.5, $p=0.010$ ) (Table S-3 in supplemental material). The optic nerve appeared to be a main target in the immune attack associated with MOG-ab because 38/41 (93\%) recurrences seen in patients with MOG-ab were ON (the optic nerve to spinal cord ratio involvement was 12.7:1). Moreover, in the group of NMO and MOG-ab, 9/11 (82\%) relapses were $\mathrm{ON}$, which was similar to the frequency seen in seronegative NMO patients $8 / 11(73 \%)(p=1.0)$. By contrast, in AQP4-ab-positive NMO patients ON occurred in 37/77 $(48 \%)$ relapses $(p=0.052)$.

\section{Serological characteristics of MOG-ab positive patients and clinical course}

In 10 out of 19 patients with MOG-ab the initial serum or CSF samples were collected at disease onset and in the other nine patients during remission after a median of 74 months from onset (range 8-324 months; IQR 12.5-180) (Supplementary Figure S-2). The antibody titres were similar regardless of the time of sample collection, clinical phenotype, or subsequent disease course (relapsing vs. monophasic course). Follow-up samples were obtained in 14/19 (73.6\%) patients with MOG-ab (including the two patients with double seropositivity) after a median follow-up of 23 months (range 5-120 months; IQR 11.8-89.5; five patients $\geq 6$ years). MOG-ab titres remained unchanged in six patients, decreased at least two dilutions in six, and became negative in two (in a case with ADEM 6 months after disease onset, and in a case with NMO 1 year after disease onset). The samples from the five patients with more than 5 years of follow-up remained antibody positive (median titre, 1:640, range 1:320-1:640). The decrease of MOG-ab titres was not associated with a monophasic course or better outcome $(p=0.1)$. Paired serum and CSF samples were available from eight patients with MOG-ab; antibodies were found only in serum of three patients (one NMO and two ON) and in serum and CSF of the other five (three NMO, one LETM, and one ON). Two of these five patients had values suggestive of intrathecal synthesis ${ }^{23}$ of MOG-ab and another patient of both MOG and AQP4-ab. In one NMO patient with a 10-year follow-up, MOG-ab were still positive in the serum (1:320), while CSF was negative (supplementary eFigure 2).

\section{Discussion}

In this series we show that $10 \%$ of all adults with demyelinating syndromes suspected to be related to AQP4-ab had MOG-ab, and that the frequency of MOG-ab went up to $15 \%$ when all patients without AQP4-ab were considered. Characterization of the underlying immune response is important because compared with patients with AQP4-ab, those with MOG-ab were younger, more frequently had a monophasic disease, and were associated with a more 
benign outcome. Moreover, irrespective of the clinical syndrome, patients with MOG-ab were less disabled and most of them were free of medication 5 years after disease onset.

ON was the more frequent syndrome associated with MOG-ab, emphasizing that the optic nerve is a main target involved in the immune attack. ${ }^{11-13}$ Six of seven patients with isolated ON had a relapsing course, whereas a monophasic course was seen in the single case who developed bilateral simultaneous ON. ${ }^{13}$ A previous study described MOG-ab in five patients with $\mathrm{ON}$; all of them had recurrent $\mathrm{ON}$ with a comparable frequency of relapses (median three, range 3-16) but the clinical characteristics were not provided. ${ }^{12}$ In the current study, despite the severity of the $\mathrm{ON}$ at symptom onset and the high frequency of relapses, patients had minimal visual acuity impairment after a median follow-up of 96 months, supporting the idea of a better recovery associated with the presence of MOG-ab.

We identified AQP4-ab in only $19 \%$ of patients with LETM; this is in contrast with the frequencies of $58 \%$ and $89 \%$ reported in two studies by other investigators using a similar sensitive method of detection. ${ }^{24,25}$ These discordant figures are likely explained by selection bias. In the first study, ${ }^{24} 32 \%$ (14/44) of AQP4-ab positive patients had history of ON or Devic's phenotype at disease onset. Our study, however, only included patients with isolated LETM. In the second study, ${ }^{25}$ all 48 patients had recurrent episodes of LETM, a feature associated with AQP4-ab. In contrast, in our series only $46 \%$ of the patients had a relapsing course. Although one could infer that the lack of relapses provides an explanation for the better outcome of patients with MOG-ab compared with those with AQP4-ab, ${ }^{11-13}$ other underlying factors might be involved given that seronegative LETM patients in our study had a worse prognosis despite $60 \%$ being monophasic. Moreover, the severity of the symptoms observed during the episode of LETM (EDSS nadir score) was similar regardless of the serological status; this finding and the fact that the three groups of patients were treated with similar immunotherapy and plasma exchange suggest that MOG-ab associate with better recovery.

Previous reports suggested that the occurrence of simultaneous ON and LETM along with a monophasic course is characteristic of NMO associated with MOG-ab. ${ }^{11,13}$ However, our experience here shows that only one of the four NMO patients with MOG-ab had these clinical features, and simultaneous ON and LETM along with a monophasic course were also found in 1/9 NMO patients with AQP4-ab and 1/2 seronegative NMO patients.

In addition to the above-discussed clinical associations, our study provides several novel concepts related to antibody titres: (1) the titres of MOG-ab were similar in patients with different syndromes (NMO, LETM, or ON), and did not differ substantially in patients with monophasic or relapsing disease, or in samples obtained at symptom presentation or during remission; (2) detection of MOG-ab can persist for several years (e.g. > 5 years) while patients are free of relapses; (3) MOG-ab seems to occur more frequently in serum than in CSF; (4) intrathecal synthesis of MOG-ab appears infrequent and may not be associated with a different outcome; and (5) the coexistence of MOG-ab and AQP4-ab in only one paediatric and two adult patients with $\mathrm{NMO}$ of our series, and a previously reported case with $\mathrm{NMO},{ }^{12}$ indicate that this association is very rare. 
Overall, MOG-ab identify a subgroup of patients with NMO or suspected limited forms of the disease who have better outcome than AQP4-ab positive patients or seronegative patients. Although the percentage of patients with MOG-ab is small, their recognition has important clinical and prognostic implications.

\section{Supplementary Material}

Refer to Web version on PubMed Central for supplementary material.

\section{Acknowledgement}

The authors thank Eva Caballero for her excellent technical support, and the neurologists of the Spanish Group of Neuromyelitis optica and Red Española de Esclerosis Múltiple.

Funding This work was supported in part by grant from La Marató de TV3 (AS; 101610), Red Española de Esclerosis Múltiple, Fondo Europeo de Desarrollo Regional (FEDER), Unión Europea, Una forma de hacer Europa (AS; RD12/0032/0002); NIH RO1NS07785 (JD), RO1CA89054 (JD), a McKinght Neurosciences of Brain Disorders award (JD), Fondo de Investigaciones Sanitarias (FIS; Spain, 11/01780; JD), Fundació la Marató de TV3 (JD) and the Fonds zur Foerderung der wissenschaftlichen Forschung (FWF), Austria, Projects J3230 (RH) and I916 (ERA-Net ERare project EDEN, MR).

\section{References}

1. Wingerchuk DM, Lennon VA, Lucchinetti CF, et al. The spectrum of neuromyelitis optica. Lancet Neurol. 2007; 6:805-815. [PubMed: 17706564]

2. Waters PJ, McKeon A, Leite MI, et al. Serologic diagnosis of NMO: A multicenter comparison of aquaporin-4-IgG assays. Neurology. 2012; 78:665-671. [PubMed: 22302543]

3. Jiao Y, Fryer JP, Lennon VA, et al. Updated estimate of AQP4-IgG serostatus and disability outcome in neuromyelitis optica. Neurology. 2013; 81:1197-1204. [PubMed: 23997151]

4. Marignier R, Bernard-Valnet R, Giraudon P, et al. Aquaporin-4 antibody-negative neuromyelitis optica: Distinct assay sensitivity-dependent entity. Neurology. 2013; 80:2194-2200. [PubMed: 23658379]

5. Reindl M, Di Pauli F, Rostasy K, et al. The spectrum of MOG autoantibody-associated demyelinating diseases. Nat Rev Neurol. 2013; 9:455-461. [PubMed: 23797245]

6. Pröbstel AK, Dornmair K, Bittner R, et al. Antibodies to MOG are transient in childhood acute disseminated encephalomyelitis. Neurology. 2011; 77:580-588. [PubMed: 21795651]

7. Di Pauli F, Mader S, Rostasy K, et al. Temporal dynamics of anti-MOG antibodies in CNS demyelinating diseases. Clin Immunol. 2011; 138:247-254. [PubMed: 21169067]

8. Rostásy K, Reindl M. Role of autoantibodies in acquired inflammatory demyelinating diseases of the central nervous system in children. Neuropediatrics. 2013; 44:297-301. [PubMed: 24166146]

9. Rostásy K, Mader S, Hennes E, et al. Persisting myelin oligodendrocyte glycoprotein antibodies in aquaporin-4 antibody negative pediatric neuromyelitis optica. Mult Scler. 2013; 19:1052-1059. [PubMed: 23257621]

10. Rostásy K, Mader S, Schanda K, et al. Anti-myelin oligodendrocyte glycoprotein antibodies in pediatric patients with optic neuritis. Arch Neurol. 2012; 69:752-756. [PubMed: 22371853]

11. Kitley J, Waters P, Woodhall M, et al. Neuromyelitis optica spectrum disorders with aquaporin-4 and myelin-oligodendrocyte glycoprotein antibodies: A comparative study. JAMA Neurol. 2014; 71:276-283. [PubMed: 24425068]

12. Mader S, Gredler V, Schanda K, et al. Complement activating antibodies to myelin oligodendrocyte glycoprotein in neuromyelitis optica and related disorders. J Neuroinflammation. 2011; 8:184. [PubMed: 22204662]

13. Sato DK, Callegaro D, Lana-Peixoto MA, et al. Distinction between MOG antibody-positive and AQP4 antibody-positive NMO spectrum disorders. Neurology. 2014; 82:474-481. [PubMed: 24415568] 
14. Wingerchuk DM, Hogancamp WF, O'Brien PC, et al. The clinical course of neuromyelitis optica (Devic's syndrome). Neurology. 1999; 53:1107-1114. [PubMed: 10496275]

15. Wingerchuk DM, Lennon VA, Pittock SJ, et al. Revised diagnostic criteria for neuromyelitis optica. Neurology. 2006; 66:1485-1489. [PubMed: 16717206]

16. Sepulveda M, Blanco Y, Rovira A, et al. Analysis of prognostic factors associated with longitudinally extensive transverse myelitis. Mult Scler. 2013; 19:742-748. [PubMed: 23037550]

17. Sellner J, Boggild M, Clanet M, et al. EFNS guidelines on diagnosis and management of neuromyelitis optica. Eur J Neurol. 2010; 17:1019-1032. [PubMed: 20528913]

18. Blanco Y, Hankiewicz K, Llufriu S, et al. Clinical spectrum associated with aquaporin-4 antibodies (NMO-IgG). Neurologia. 2010; 25:5-12. [PubMed: 20388455]

19. Krupp LB, Tardieu M, Amato MP, et al. International Pediatric Multiple Sclerosis Study Group criteria for pediatric multiple sclerosis and immune-mediated central nervous system demyelinating disorders: Revisions to the 2007 definitions. Mult Scler. 2013; 19:1261-1267. [PubMed: 23572237]

20. McDonald WI, Compston A, Edan G, et al. Recommended diagnostic criteria for multiple sclerosis: Guidelines from the International Panel on the diagnosis of multiple sclerosis. Ann Neurol. 2001; 50:121-127. [PubMed: 11456302]

21. Höftberger R, Sabater L, Marignier R, et al. An optimized immunohistochemistry technique improves NMO-IgG detection: Study comparison with cell-based assays. PLoS One. 2013; 8:e79083. [PubMed: 24223884]

22. Titulaer M, Höftberger R, Izuka T, et al. Overlapping demyelinating syndromes and anti-NMDA receptor encephalitis. Ann Neurol. 2014; 75:411-428. [PubMed: 24700511]

23. Saiz A, Blanco Y, Sabater L, et al. Spectrum of neurological syndromes associated with glutamic acid decarboxylase antibodies: Diagnostic clues for this association. Brain. 2008; 131:2553-2563. [PubMed: 18687732]

24. Kitley J, Leite MI, Kuker W, et al. Longitudinally extensive transverse myelitis with and without aquaporin 4 antibodies. JAMA Neurol. 2013; 70:1375-1381. [PubMed: 23999580]

25. Jiao Y, Fryer JP, Lennon VA, et al. Aquaporin 4 IgG serostatus and outcome in recurrent longitudinally extensive transverse myelitis. JAMA Neurol. 2014; 71:48-54. [PubMed: 24248262] 
Table 1

Comparison of demographic and clinical features between seropositive (MOG-ab or AQP4-ab) and seronegative patients.

\begin{tabular}{|c|c|c|c|c|c|}
\hline Patients characteristics $n=174^{a}$ & MOG-ab+ $n=17(\%)$ & $\begin{array}{c}\mathrm{AQP4}-\mathrm{ab}+n \\
(\%)\end{array}$ & Seronegative $n=96(\%)$ & $p$-value $b$ & $p$-value ${ }^{c}$ \\
\hline \multicolumn{6}{|l|}{ Phenotype } \\
\hline $\operatorname{NMO}(46)^{a}$ & $4(24)$ & $37(63)$ & $5(5)$ & 0.006 & 0.028 \\
\hline LETM (84) & $5(29)$ & $16(27)$ & $63(66)$ & 1.00 & 0.007 \\
\hline ON (39) & $7(41)$ & $6(10)$ & $26(27)$ & 0.007 & 0.256 \\
\hline $\operatorname{ADEM}(3)$ & $1(6)$ & 0 & $2(2)$ & 0.224 & 0.389 \\
\hline Female, $n(\%)$ & $9(53)$ & $53(90)$ & $63(66)$ & 0.002 & 0.412 \\
\hline Age at onset, $y$, median (range) & $27(18-59)$ & $40.5(18-73.5)$ & $37.5(18-76.5)$ & 0.017 & 0.021 \\
\hline Coexisting autoimmune disorders, $n(\%)$ & $1(6)$ & $9 / 56(16)$ & 9/77 (11.5) & 0.435 & 1.00 \\
\hline $\begin{array}{l}\text { Simultaneous ON+myelitis attacks at onset, } \\
n(\%)\end{array}$ & $3(18)$ & $9(15)$ & $2(2)$ & 0.811 & 0.024 \\
\hline Nadir EDSS score, median (range) & $4.0(3.0-8.0)$ & $6.0(2.0-8.0)$ & $5.0(3.0-9.0)$ & 0.782 & 0.623 \\
\hline \multicolumn{6}{|l|}{ MRI brain classification } \\
\hline Normal & $13(76)$ & $31 / 57(54)$ & $52 / 81(64)$ & 0.159 & 0.407 \\
\hline Nonspecific & $4(24)$ & $22 / 57(39)$ & 27/81(33) & 0.386 & 0.570 \\
\hline MS-like & 0 & $4 / 57(7)$ & 2/81 (3) & 0.568 & 1.00 \\
\hline \multicolumn{6}{|l|}{ MRI cord lesion } \\
\hline Median vertebral segments (range) & $5(3-21)$ & $5(2-23)$ & $6(3-23)$ & 0.674 & 0.991 \\
\hline \multicolumn{6}{|l|}{ CSF } \\
\hline $\mathrm{WBC}>50 / \mathrm{mm}^{3}, n(\%)$ & $4 / 16(25)$ & $4 / 39(10)$ & $11 / 64(17)$ & 0.211 & 0.485 \\
\hline OCBs, $n(\%)$ & $1(6)$ & $5 / 44(11)$ & $10 / 62(16)$ & 1.00 & 0.445 \\
\hline ANA, $n(\%)$ & $6(35)$ & $29(49)$ & $44 / 92(48)$ & 0.408 & 0.431 \\
\hline \multicolumn{6}{|l|}{ Acute treatment } \\
\hline IVMP + PE, $n(\%)$ & $4(24)$ & $11(19)$ & $13 / 86(15)$ & 0.732 & 0.474 \\
\hline Monophasic course, $n(\%)$ & $7(41)$ & $4 / 57(7)$ & $54(56)$ & 0.002 & 0.297 \\
\hline No. of attacks, median (range) & $3(1-14)$ & $3(1-14)$ & $2(1-18)$ & 0.743 & 0.139 \\
\hline Time to first relapse, mo, median (range) & $7.5(1-160)$ & $8(1-144)$ & $6(1-123)$ & 0.989 & 0.650 \\
\hline Chronic treatment, $n(\%)$ & $6(35)$ & 49/54 (91) & $34 / 92(37)$ & $<0.001$ & 1.00 \\
\hline Last EDSS, median (range) & $1.5(0-3.0)$ & $4.0(0-10)$ & $3.0(0-8.5)$ & $<0.001$ & $<0.001$ \\
\hline Follow-up, mo, median (range) & $67(11-415)$ & $65(1-355)$ & $30(1-175)$ & 0.812 & 0.010 \\
\hline
\end{tabular}

MOG-ab: antibodies to myelin oligodendrocyte glycoprotein; AQP4-ab: aquaporin 4 antibodies; NMO: neuromyelitis optica; LETM: longitudinal extensive transverse myelitis; ON: optic neuritis; ADEM: acute disseminated encephalomyelitis; EDSS: Expanded Disability Status Scale; CSF: cerebrospinal fluid; WBC: white blood cells; OCBs: oligoclonal bands; ANA: antinuclear antibody; IVMP: intravenous methylprednisolone; PE: plasma exchange.

${ }^{a}$ Two NMO patients with both MOG-ab and AQP4-ab were excluded from analysis.

${ }^{b}$ Comparison between MOG-ab+ and AQP4-ab+ patients;

${ }^{c}$ Comparison between MOG-ab+ and seronegative patients.

Mult Scler. Author manuscript; available in PMC 2016 April 08. 


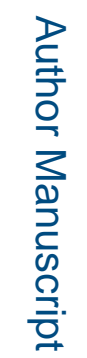

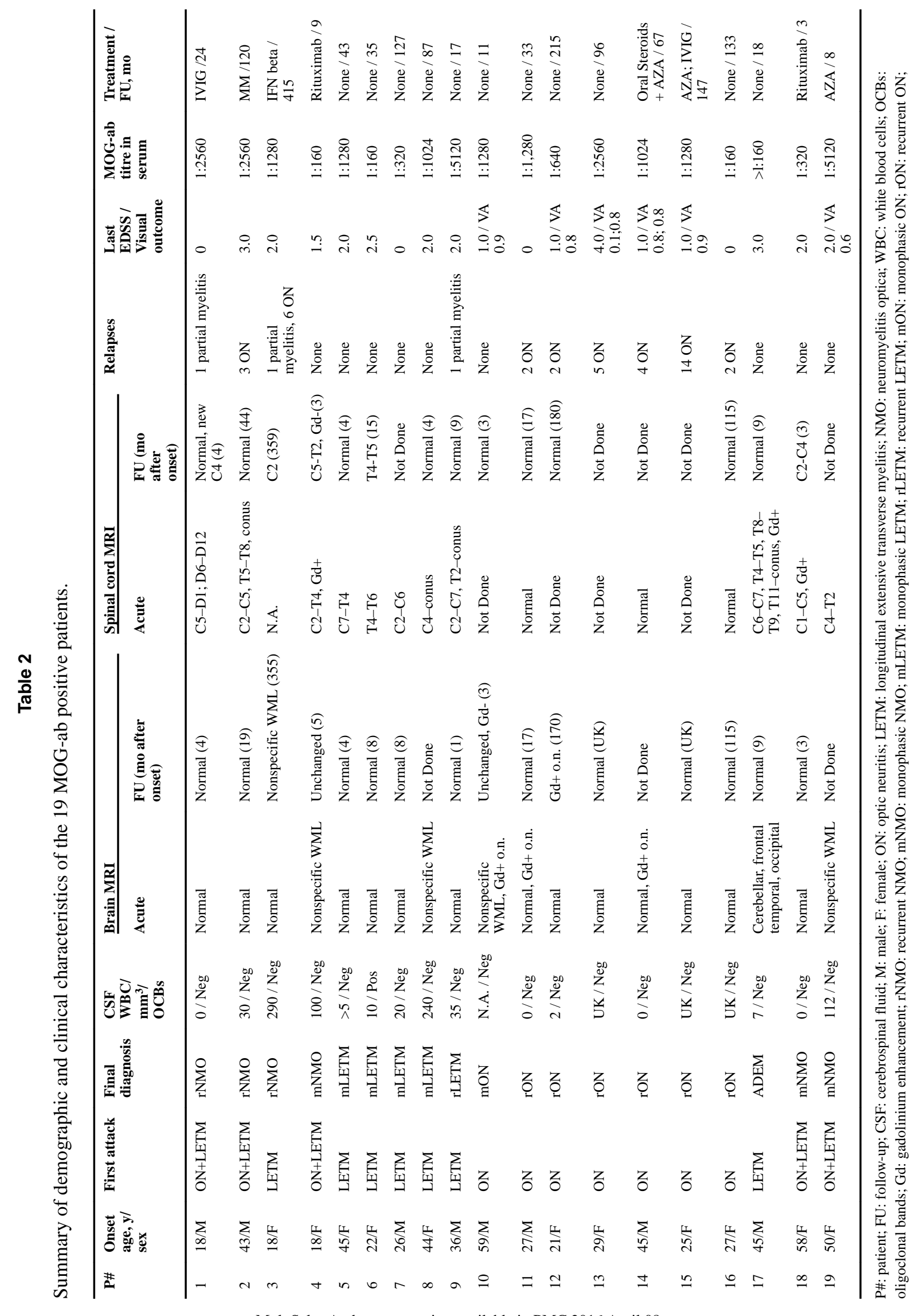

Mult Scler. Author manuscript; available in PMC 2016 April 08. 


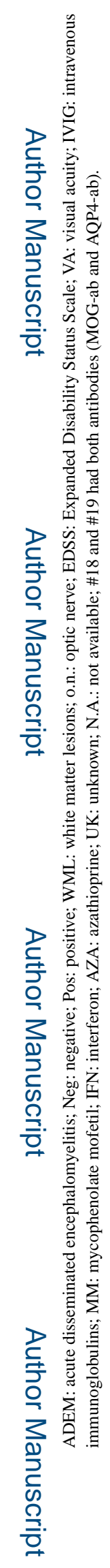

